\title{
Fluorescence Detection of Zabofloxacin, a Novel Fluoroquinolone Antibiotic, in Plasma, Bile, and Urine by HPLC: The First Oral and Intravenous Applications in a Pharmacokinetic Study of Rats
}

\author{
Hyo-Eon Jin, In-Hyul Kang, and Chang-Koo Shim \\ National Research Laboratory for Transporters Targeted Drug Design, Research Institute of Pharmaceutical Sciences, \\ College of Pharmacy, Seoul National University, Seoul, 151-742, Republic of Korea.
}

Received, May 15, 2011; Revised, June 14, 2011; Accepted, July 12, 2011; Published, July 15, 2011.

\begin{abstract}
Purpose: To develop an HPLC method using fluorescence detection for the pharmacokinetic evaluation of levels of zabofloxacin, a novel broad-spectrum fluoroquinolone antibiotic, in the plasma, bile and urine of rats. Methods: A simple reversed-phase HPLC method using a $\mathrm{C}_{18}$ column with fluorescence detection was developed and validated for the simultaneous determination of zabofloxacin and enrofloxacin as an internal standard. The plasma sample was treated with methanol for protein precipitation, and treatment of the bile and urine samples included deproteinization and extraction using chloroform. The applicability of the developed assay method to pharmacokinetic studies of zabofloxacin in rats was examined. Zabofloxacin was intravenously and orally administered to rats at a dose of $20 \mathrm{mg} / \mathrm{kg}$. Results: The limit of quantification (LOQ) was determined to be $0.05 \mu \mathrm{g} / \mathrm{mL}$ for the plasma with acceptable linearity ranging from 0.05 to $25 \mu \mathrm{g} / \mathrm{mL}$ ( $\mathrm{R}>0.999)$, and $0.5 \mu \mathrm{g} / \mathrm{mL}$ for the bile and urine samples with acceptable linearity ranging from 0.5 to $100 \mu \mathrm{g} / \mathrm{mL}(\mathrm{R}>0.999)$. The validation parameters for zabofloxacin were found to be acceptable according to FDA assay validation (2001). While zabofloxacin in plasma and urine has been stable in all tested handling conditions, it has been unstable in bile during freeze-thaw cycles for $24 \mathrm{~h}$ at room temperature. Following intravenous and oral administration of zabofloxacin to rats at a dose of 20 $\mathrm{mg} / \mathrm{kg}$, concentration was quantifiable in plasma for up to $8 \mathrm{~h}$. The bioavailability of zabofloxacin was $27.7 \%$, and it was excreted into bile and urine at about $7 \%$ and $9 \%$ each per oral administration. Conclusions: These observations suggest that a validated assay can be used in pharmacokinetic studies of zabofloxacin in small animals. Due to the limited stability of zabofloxacin in rat bile, freeze-thaw cycles or prolonged handling at room temperature is not recommended.
\end{abstract}

This article is open to POST-PUBLICATION REVIEW. Registered readers (see "For Readers") may comment by clicking on ABSTRACT on the issue's contents page.

\section{INTRODUCTION}

Quinolones comprise a family of synthetic broad-spectrum antibiotics. The first generation of quinolones began with the introduction of nalidixic acid in 1962 for the treatment of urinary tract infections in humans (1). The majority of quinolones in clinical use belong to the subset fluoroquinolones, which have a fluorine atom attached to the central ring system, typically at the 6 or the C-7 position. Fluoroquinolones comprise a class of antibiotics that are of great significance in the field of antibacterial chemotherapy due to their remarkably broad spectrum of activity (2). However, as the use of fluoroquinolones has increased, a continual increase in bacterial resistance to these drugs has been widely recognized (3-4). Therefore, the development of new antibacterial substances that are effective even against drug-resistant bacteria is a matter of utmost importance.

Zabofloxacin (DW-224a, Fig. 1A) is a new fluoroquinolone that was developed by Dong Wha Pharmaceuticals (Seoul, Korea). It is a potent and selective inhibitor of the essential bacterial Type II topoisomerases (DNA gyrase) and topoisomerase IV, which are involved in DNA replication and metabolism (5). Zabofloxacin has broad-spectrum antibacterial activity with enhanced activity against Gram-positive and Gram-negative organisms, particularly $S$. pneumoniae including pneumococcus strains that are resistant to other fluoroquinolones (6).

Corresponding Author: Chang-Koo Shim, Ph.D. National Research Laboratory for Transporters Targeted Drug Design, Research Institute of Pharmaceutical Sciences, College of Pharmacy, Seoul National University, Gwanak-gu, Seoul , Korea; E-mail: shimck@snu.ac.kr 
The overall antibacterial activity of zabofloxacin against gram-positive pathogens, such as methicillin-resistant Staphylococcus aureus (MRSA), methicillin-resistant coagulase-negative staphylococci, S. pneumoniae, S.pyogenes, and E. faecalis, was more potent than the activity of reference compounds, such as ciprofloxacin, moxifloxacin, and gemifloxacin (7). Therefore, zabofloxacin, with its expanded anti-pneumococcal activity, is expected to be highly effective for the treatment of community-acquired respiratory tract infections, urinary tract infections, septicemia, systemic infections, skin and soft tissue infections, bacteremia, otitis media, and possibly endocarditis (8-10).

Although zabofloxacin has much clinical utility, reports of quantitative methods for biological fluids (i.e., plasma, bile, and urine) and pharmacokinetic studies have been insufficient. The LC-MS methods for determination of zabofloxacin in plasma have already been described by this group (11). Zabofloxacin in rat plasma was analyzed by selected ion monitoring (SIM) at $\mathrm{m} / \mathrm{z}$ transitions of 402 and the lower limit of quantification (LLOQ) was determined to be $10 \mathrm{ng} / \mathrm{mL}$, with acceptable linearity ranging from 10 to $5,000 \mathrm{ng} / \mathrm{mL}$ ( $\mathrm{R}>$ 0.999) (11). However, LC-MS equipment is expensive and not universally available. Fluorescence detection is considered to be simple and accessible by the research community. Moreover, fluorescence detection often improves analytical sensitivity compared with UV detection, and fluorescence detection was tried in this investigation.

The objective of the present study was to develop and validate an HPLC method to assay zabofloxacin in rat plasma, bile and urine that would be applicable to routine pharmacokinetics of zabofloxacin following intravenous and oral administration. To the best of our knowledge this is the first report to validate zabofloxacin in the bile and urine and to assess its pharmacokinetics following both intravenous and oral administration in rats.

\section{METHODS}

\section{Materials}

Zabofloxacin hydrochloride $(99.0 \%$ purity) was provided by Dong Wha Pharmaceuticals Co. (Seoul, Korea). Zabofloxacin (Mw 437.9) (Fig. 1A) has a melting point of $155^{\circ} \mathrm{C}$, and is weakly acidic. Enrofloxacin (purity $\geq 98 \%$, Fig. 1B), which served as the internal standard (IS) in this assay, was purchased from Sigma-Aldrich (St. Louis, MO, USA). Chloroform was purchased from Daejung Chemicals Co. (Shihung, Korea). Acetonitrile and methanol were purchased from Fischer Scientific (NJ, USA).<smiles>CO/N=C1\CN(c2nc3c(cc2F)c(=O)c(C(=O)O)cn3C2CC2)CC12CNC2</smiles>

(B)

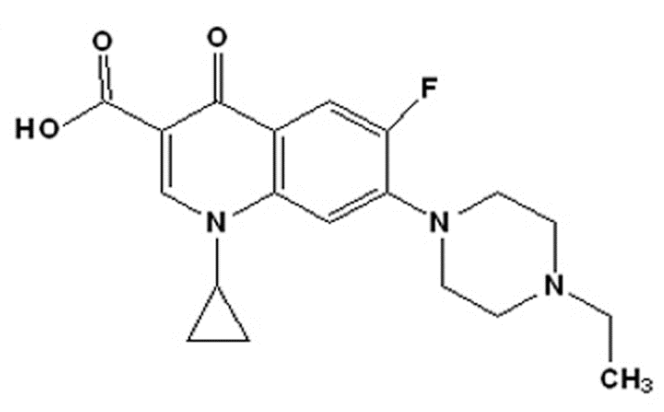

Figure 1. Chemical structure of (A) zabofloxacin and (B) enrofloxacin

\section{Animals}

Male Sprague-Dawley (SD) rats (Orient Bio Inc., Seong-Nam, Korea) weighing 240-310 g (i.e., 7-8 weeks of age) were used for the in vivo pharmacokinetic studies. The SD rats were housed in a temperature- $\left(20-23^{\circ} \mathrm{C}\right)$ and humidity-controlled (50-60\%) room under a constant 12-h light/dark cycle. The SD rats were given water and standard rat chow ad libitum. All animal experiments were performed in accordance with the Guidelines for Animal Care and Use, Seoul National University.

\section{Instrumental and Chromatographic Conditions}

The chromatography device was equipped with an HPLC system (Yong Lin Co. Ltd.), and used a Midas Autosampler and an SP930D pump. The separation of zabofloxacin and the IS from endogenous substances was performed using a Gemini-NX $\mathrm{C}_{18}$ column [150 mm length $\times 4.6 \mathrm{~mm}$ 
internal diameter, $5 \mu \mathrm{m}$ (Phenomenex, USA)] and a SecurityGuard $^{\mathrm{TM}}[4.0 \mathrm{~mm}$ length $\times 3.0 \mathrm{~mm}$ internal diameter (Phenomenex, USA)].

Mixtures of acetonitrile and a $20 \mathrm{mM}$ potassium phosphate buffer $(\mathrm{pH} 2.4), 19: 81(\mathrm{v} / \mathrm{v})$ for rat plasma and 17.5:82.5 (v/v) for bile and urine, were used as the mobile phase. The mobile phase was filtered through a $0.45 \mu \mathrm{m}$ nylon filter (Whatman Inc., Haverhill, MA) and degassed in an ultrasonic bath for $30 \mathrm{~min}$ prior to use. The flow rate of the mobile phase was maintained at $1 \mathrm{~mL} / \mathrm{min}$. A Jasco FP-2020 plus fluorescence detector was operated at an excitation wavelength of $263 \mathrm{~nm}$ and an emission wavelength of $410 \mathrm{~nm}$.

\section{Preparation of the Standard Solution}

For the analysis of zabofloxacin in rat plasma, a stock solution of zabofloxacin $(0.5 \mathrm{mg} / \mathrm{mL})$ was prepared by dissolving zabofloxacin in methanol. Standard solutions were then prepared by serial dilution of the stock solution with methanol to obtain the desired concentration. A stock solution of enrofloxacin (1 $\mathrm{mg} / \mathrm{mL}$ in methanol) was prepared and diluted with methanol to use the IS solutions. All solutions were stored at $-80^{\circ} \mathrm{C}$ until further use in analysis. Plasma standards of zabofloxacin were prepared by mixing $50 \mu \mathrm{L}$ of each standard solution with $50 \mu \mathrm{L}$ of blank rat plasma in Eppendorf tubes to give final zabofloxacin concentrations of 50, 100, 500, 1000, 5000,10000 , and $25000 \mathrm{ng} / \mathrm{mL}$ in plasma. An aliquot $(50 \mu \mathrm{L})$ of an IS solution $(1 \mu \mathrm{g} / \mathrm{mL})$ was then added to each plasma standard. After vortex-mixing and centrifugation at $16,000 \mathrm{~g}$ for 3 min, a $50 \mu \mathrm{L}$ aliquot was injected directly into the HPLC device.

For the analysis of zabofloxacin in rat bile and urine, a stock solution of zabofloxacin $(20 \mathrm{mg} / \mathrm{mL})$ was prepared by dissolving zabofloxacin in DDW. Standard solutions were then prepared by serial dilution of the stock solution with DDW to obtain the desired concentration. A stock solution of enrofloxacin $(200 \mu \mathrm{g} / \mathrm{mL}$ in DDW) was prepared and diluted with DDW to use the IS solutions. All solutions were stored at $-80^{\circ} \mathrm{C}$ until further use in analysis. Bile and urine standards of zabofloxacin were prepared by mixing $10 \mu \mathrm{L}$ of each standard solution with $50 \mu \mathrm{L}$ of blank rat bile or urine in Eppendorf tubes to give final zabofloxacin concentrations of $0.5,1,5,10,25,50$, and 100 $\mu \mathrm{g} / \mathrm{mL}$ in bile or urine. An aliquot $(10 \mu \mathrm{L})$ of an IS solution $(75 \mu \mathrm{g} / \mathrm{mL})$ was then added to each bile and urine standard and mixed with $500 \mu \mathrm{L}$ of chloroform to precipitate the proteins and extract the drug in the bile and urine samples. After vortex mixing for $1 \mathrm{~min}$ and centrifuging at $16,000 \mathrm{~g}$ for 3 min, a $450 \mu \mathrm{L}$ aliquot of the chloroform layer was transferred to a clean tube and mixed with $200 \mu \mathrm{L}$ of $20 \mathrm{mM}$ phosphate buffer. After vortex mixing for $1 \mathrm{~min}$ and centrifuging at $16,000 \mathrm{~g}$ for $3 \mathrm{~min}$, a $50 \mu \mathrm{L}$ aliquot of the $20 \mathrm{mM}$ phosphate buffer layer was injected into the HPLC system.

\section{Treatment of Plasma, Urine, and Bile Samples}

For the analysis of zabofloxacin in the plasma, 50 $\mu \mathrm{L}$ of the plasma sample was spiked with $50 \mu \mathrm{L}$ of IS $(1 \mu \mathrm{g} / \mathrm{mL}$ enrofloxacin in methanol) and mixed with $50 \mu \mathrm{L}$ of acetonitrile to precipitate plasma proteins. After vortex mixing for $3 \mathrm{~min}$ and centrifuging at $16,000 \mathrm{~g}$ for $3 \mathrm{~min}$, a $50 \mu \mathrm{L}$ aliquot of the clear supernatant (plasma sample) was injected into the HPLC system.

For the analysis of zabofloxacin in bile, $50 \mu \mathrm{L}$ of a bile sample was spiked with a $10 \mu \mathrm{L}$ IS (75 $\mu \mathrm{g} / \mathrm{mL}$, enrofloxacin in DDW) solution and mixed with $500 \mu \mathrm{L}$ of chloroform to precipitate the proteins and extract the drug from the bile sample. After vortex mixing for $1 \mathrm{~min}$ and centrifuging at $16,000 \mathrm{~g}$ for $3 \mathrm{~min}$, a $450 \mu \mathrm{L}$ aliquot of the chloroform layer was transferred to a clean tube and mixed with $200 \mu \mathrm{L}$ of $20 \mathrm{mM}$ phosphate buffer. After vortex mixing for $1 \mathrm{~min}$ and centrifuging at $16,000 \mathrm{~g}$ for $3 \mathrm{~min}$, a $50 \mu \mathrm{L}$ aliquot of the $20 \mathrm{mM}$ phosphate buffer layer (bile sample) was injected into the HPLC system.

For the analysis of zabofloxacin in the urine, 50 $\mu \mathrm{L}$ of a urine sample was spiked with $10 \mu \mathrm{L}$ of the IS $(75 \mu \mathrm{g} / \mathrm{mL}$, enrofloxacin in DDW) solution and mixed with $500 \mu \mathrm{L}$ of chloroform to precipitate the proteins and extract the drug in the urine sample. After vortex mixing for $3 \mathrm{~min}$ and centrifuging at $16,000 \mathrm{~g}$ for $3 \mathrm{~min}$, a $450 \mu \mathrm{L}$ aliquot of the chloroform layer was transferred to a clean tube and mixed with $200 \mu \mathrm{L}$ of $20 \mathrm{mM}$ phosphate buffer. After vortex mixing for $1 \mathrm{~min}$ and centrifuging at $16,000 \mathrm{~g}$ for $3 \mathrm{~min}$, a $50 \mu \mathrm{L}$ aliquot of the $20 \mathrm{mM}$ phosphate buffer layer (urine sample) was injected into the HPLC system.

\section{Validation Procedures}

A complete validation for the assay of zabofloxacin in rat serum was performed in accordance with USFDA guidelines (12). Specificity was assessed by analysis of serum from 5 different rats to determine the absence of endogenous substances with retention times similar to those of zabofloxacin and the IS.

The LOQ was defined as a concentration with a 
precision of less than $20 \%$ of the relative standard deviation (RSD) and an accuracy of between 80 and $120 \%$ of the theoretical value. The signal-to-noise ratio for the LOQ sample was confirmed to be not less than 10 (12).

The linearity of the assay was assessed using the zabofloxacin standard samples over a concentration range of $0.05-25 \mu \mathrm{g} / \mathrm{mL}$ for plasma and $0.5-100$ $\mu \mathrm{g} / \mathrm{mL}$ for bile and urine. Calibration curves were constructed using the ratios of the zabofloxacin peak areas to that of the IS in the plasma standards. The linearity of the calibration curve was determined using linear regression analysis. A calibration curve with a correlation coefficient of not less than 0.995 was considered to be linear (12).

The intraday and interday precision and accuracy of the method were determined by analyzing 5 sets each of LOQ, low-quality control (LQC), mid-quality control (MQC), and highquality control (HQC) on 5 different days. Concentrations of LOQ, LQC, MQC, and HQC were $0.05,0.5,5$, and $25 \mu \mathrm{g} / \mathrm{mL}$ for plasma, and 0.5 , 5, 25, and $100 \mu \mathrm{g} / \mathrm{mL}$ for bile and urine, respectively. For the intraday validation, five sets of QC samples with concentration levels were assayed in 1 day. For interday validation, 5 sets of QC samples were determined on 5 different days. Precision was expressed as a percentage of the relative standard deviation (RSD) with the acceptance criteria of RSD $\pm 15 \%$ at each concentration with the exception of LOQ, which was allowed RSD $\pm 20 \%$. Accuracy percentage was expressed as [(calculated amount / theoretical concentration $) \times 100$ ] with acceptance set at $\pm 15 \%$ of the nominal concentrations of QC samples except at LOQ where it was set at $\pm 20 \%$. The recoveries of zabofloxacin were calculated by comparing the observed concentration with the spiked concentrations.

Stability studies for zabofloxacin in plasma, bile and urine included freeze/thaw stability, bench-top stability (at room temperature for $24 \mathrm{hr}$ ), and long-term stability $\left(-80^{\circ} \mathrm{C}\right.$ for 1 week), which were performed at LQC, MQC, and HQC. Freeze/thaw stability was tested after 3 freeze/thaw cycles spaced at least $24 \mathrm{hr}$ apart with sample storage at $80^{\circ} \mathrm{C}$ between each thaw. The results obtained for the samples stored at room temperature and in a refrigerator were compared with those of freshly prepared samples.

\section{Application to Pharmacokinetic Studies of Zabofloxacin}

The applicability of the developed assay method to pharmacokinetic studies of zabofloxacin in rats was examined. Rats $(\mathrm{n}=4)$ were anesthetized with ketamine $(45 \mathrm{mg} / \mathrm{kg}$, im) and acepromazine (5 $\mathrm{mg} / \mathrm{kg}, \mathrm{im})$, and the femoral artery and vein were cannulated with a polyethylene tube (PE-50; Clay Adams, Parsippany, NJ, USA) filled with heparinized saline $(20 \mathrm{IU} / \mathrm{mL})$ to prevent blood clotting (13). After recovery from the anesthesia, the rats were intravenously administered zabofloxacin $(20 \mathrm{mg} / \mathrm{mL}$ in DDW $)$ at a dose of 20 $\mathrm{mg} / \mathrm{kg}$. Blood samples $(120 \mu \mathrm{L})$ were taken from the femoral artery cannula at $0,1,3,10,30,60$, $120,240,360$, and $480 \mathrm{~min}$ after intravenous administration of zabofloxacin. The blood volume withdrawn at each time point was replaced with an equal volume of saline to compensate for fluid loss. The plasma was separated by centrifugation of the whole blood at $16,000 \mathrm{~g}$ for $3 \mathrm{~min}$ and was then stored at $-80^{\circ} \mathrm{C}$ until analysis.

For oral pharmacokinetic study of zabofloxacin, the femoral artery, vein and urinary bladder were catheterized with polyethylene tubing (PE-50; Clay Adams, Parsippany, NJ, USA) for blood sampling, fluid compensation and urine collection, respectively. Rats $(n=4)$ were orally administered zabofloxacin $(20 \mathrm{mg} / \mathrm{mL}$ in DDW) at a dose of 20 $\mathrm{mg} / \mathrm{kg}$. Blood samples $(120 \mu \mathrm{L})$ were taken from the femoral artery cannula at $0,3,7,15,30,60,90$, $120,240,360$, and $480 \mathrm{~min}$ after oral administration of zabofloxacin. The blood volume withdrawn at each time point was replaced with an equal volume of saline to compensate for fluid loss. The plasma was separated by centrifugation of the whole blood at $16,000 \mathrm{~g}$ for $3 \mathrm{~min}$ and was then stored at $-80^{\circ} \mathrm{C}$ until analysis. Urine samples were collected at 0-2, 2-4, 4-6, 6-8, 8-10, and 10-24 h. At the end of the each time point, the urinary bladder was rinsed with $0.5 \mathrm{~mL}$ of normal saline and each rinsing was combined with collected urine. After measuring each urine sample was stored at $-80^{\circ} \mathrm{C}$ until analysis.

Zabofloxacin at a dose of $20 \mathrm{mg} / \mathrm{kg}$ was orally administered to rats using a feeding tube $(n=4)$ after bile duct cannulation (without cannulation of the femoral artery), and bile samples were collected during $0-1,1-2,2-4,4-6,6-8$, and 8-24 h.

The plasma, bile, and urine samples were then processed as described above in the Treatment of Plasma, Urine, and Bile Sample sections, and the concentration of zabofloxacin was analyzed by HPLC. 


\section{DATA AND STATISTICAL ANALYSIS}

The peak concentration $\left(\mathrm{C}_{\max }\right)$ and time to reach $\mathrm{C}_{\max }\left(\mathrm{T}_{\max }\right)$ were read directly from individual zabofloxacin plasma concentration-time profiles. The area under the plasma concentration-time curve $\left(\mathrm{AUC}_{0-\mathrm{t}}\right)$ was calculated using the linear trapezoidal method from 0 to $8 \mathrm{~h}$. The area under the plasma concentration-time curve from zero to time infinity $\left(\mathrm{AUC}_{\infty}\right)$ was calculated using the trapezoidal extrapolation method (14). In the extrapolation, the area from the last datum point to time infinity was estimated by dividing the last measured plasma by the terminal-phase rate constant. Standard methods (14) were used to calculate the terminal half-life $\left(\mathrm{T}_{1 / 2}\right)$ using a noncompartmental analysis program (WinNonlin ${ }^{\circledR} 3.1$, Pharsight Co., Mountain View, CA, USA). The biliary clearance $\left(\mathrm{CL}_{b}\right)$ and renal clearance $\left(\mathrm{CL}_{\mathrm{R}}\right)$ were estimated using Eq. (1) and (2), in which the cumulative amount excreted in the bile for $24 \mathrm{~h}$ $\left(\mathrm{X}_{\mathrm{b}}\right)$ and the cumulative amount excreted in the urine for $24 \mathrm{~h}\left(\mathrm{X}_{\mathrm{u}}\right)$ were experimentally measured.

$$
\begin{aligned}
& \mathrm{CL}_{\mathrm{b}}=\mathrm{X}_{\mathrm{b}} / \mathrm{AUC}_{\infty} \\
& \mathrm{CL}_{\mathrm{R}}=\mathrm{X}_{\mathrm{u}} / \mathrm{AUC}_{\infty}
\end{aligned}
$$

Data are presented as the mean $\pm \mathrm{SD}$ for all experiments.

\section{RESULTS}

\section{Assay Validation of Zabofloxacin in the Plasma}

Fig. 2 presents representative HPLC chromatograms of blank rat plasma, blank plasma spiked with zabofloxacin, and blank plasma spiked with enrofloxacin (IS). After optimizing the chromatographic separation conditions, a reproducible separation among zabofloxacin, IS, and endogenous components was obtained. The chromatogram for a blank rat plasma sample showed no interfering peaks for the retention times of the analyte and the IS (Fig. 2). The retention times for zabofloxacin and the IS were determined to be approximately 5.25 and $3.05 \mathrm{~min}$, respectively, indicating that the analysis can be completed within $7.5 \mathrm{~min}$ (Fig. 2B and 2C). The retention times for zabofloxacin and IS in the standard plasma samples were identical to those of plasma samples obtained following oral administration of the drug at a dose of $20 \mathrm{mg} / \mathrm{kg}$.

Under the analytical conditions used in the present study, the calibration curves for zabofloxacin in rat plasma were found to be linear over a concentration range of $0.05-25 \mu \mathrm{g} / \mathrm{mL}$. Using a linear least-squares regression analysis, the calibration equation for zabofloxacin was $(n=5$, the slopes and intercepts in the mean \pm standard deviation $) \mathrm{y}=(0.2565 \pm 0.0033) \mathrm{x}+(0.01574 \pm$ 0.0030 ) with $\mathrm{r}^{2}=0.9998 \pm 0.0002$, where $\mathrm{y}, \mathrm{x}, \mathrm{r}$ represent the peak area ratio, the concentration of zabofloxacin in the plasma, and the correlation coefficient, respectively.

QC samples at 4 different concentrations $(0.05$, $0.5,5$, and $25 \mathrm{ng} / \mathrm{mL}$ ) were analyzed in 5 replicates to determine the values for intraday and interday accuracy/precision. A summary of the intraday and interday accuracy/precision values is listed in Table 1. The values for intraday accuracy for zabofloxacin ranged from 95.7 to $106 \%$, while the intraday precision in RSD was less than $9.1 \%$. The values for interday accuracy for the analyte ranged from 89 to $100.0 \%$, and the precision was less than $9.5 \%$. The limit of detection (LOD) for zabofloxacin was $<0.05 \mu \mathrm{g} / \mathrm{mL}$ for the plasma sample. Based on the estimated precision and accuracy, the limit of quantification for this assay was set at $0.05 \mu \mathrm{g} / \mathrm{mL}$. These results indicated that the accuracy and precision of the current assay were within the recommendations for assay validation as stipulated in "Guidance for Industry: Bioanalytical Method Validation (12)," and that the reproducibility of the assay was adequate.

The stability of zabofloxacin in the rat plasma was assessed for typical storage and handling conditions (Table 4). After freeze-thaw cycles, the changes in the ratios of the peak areas of zabofloxacin to those of the IS were negligible (i.e., 96.9-98.7\%). In the cases of bench-top and long-term stability, the peak ratios were comparable to those of the fresh sample (i.e., 97.7$102 \%$ for bench-top stability, and $96.8-97.6 \%$ for long-term stability).

\section{Assay Validation of Zabofloxacin in the Bile}

Fig. 3 presents representative HPLC chromatograms of blank rat bile, blank bile spiked with zabofloxacin, and blank bile spiked with enrofloxacin (IS). After optimizing the chromatographic separation conditions, a reproducible separation among zabofloxacin, IS, and endogenous components was obtained. The chromatogram for a blank rat bile sample showed no interfering peaks for the retention times of the analyte and the IS (Fig. 3). 


\begin{tabular}{|c|c|c|c|c|}
\hline \multirow[t]{2}{*}{ Batch } & \multicolumn{4}{|c|}{ Theoretical concentration $(\mu \mathrm{g} / \mathrm{mL})$} \\
\hline & LOQ & LQC & MQC & HQC \\
\hline & 0.05 & 0.5 & 5 & 25 \\
\hline \multicolumn{5}{|l|}{ (A) Intraday accuracy and precision } \\
\hline Mean estimated concentration $(\mu \mathrm{g} / \mathrm{mL})$ & 0.053 & 0.48 & 5.09 & 24.8 \\
\hline Precision $(\mathrm{CV}, \%)^{\mathrm{a}}$ & 5.6 & 9.1 & 5.1 & 8.6 \\
\hline Accuracy $(\%)^{b}$ & 106 & 96 & 102 & 99 \\
\hline \multicolumn{5}{|l|}{ (B) Interday accuracy and precision } \\
\hline Mean estimated concentration $(\mu \mathrm{g} / \mathrm{mL})$ & 0.044 & 0.49 & 4.94 & 25.0 \\
\hline Precision $(\mathrm{CV}, \%)^{\mathrm{a}}$ & 9.5 & 6.4 & 4.1 & 1.5 \\
\hline Accuracy $(\%)^{\mathrm{b}}$ & 89 & 99 & 99 & 100 \\
\hline
\end{tabular}

\begin{tabular}{|c|c|c|c|c|}
\hline \multirow[t]{2}{*}{ Batch } & \multicolumn{4}{|c|}{ Theoretical concentration $(\mu \mathrm{g} / \mathrm{mL})$} \\
\hline & LOQ & LQC & MQC & HQC \\
\hline & 0.5 & 5 & 25 & 100 \\
\hline \multicolumn{5}{|l|}{ (A) Intraday accuracy and precision } \\
\hline Mean estimated concentration $(\mu \mathrm{g} / \mathrm{mL})$ & 0.51 & 5.16 & 26.0 & 105 \\
\hline Precision $(\mathrm{CV}, \%)^{\mathrm{a}}$ & 2.0 & 5.3 & 3.1 & 2.1 \\
\hline Accuracy $(\%)^{b}$ & 102 & 103 & 104 & 105 \\
\hline \multicolumn{5}{|l|}{ (B) Interday accuracy and precision } \\
\hline Mean estimated concentration $(\mu \mathrm{g} / \mathrm{mL})$ & 0.56 & 5.01 & 24.7 & 100 \\
\hline Precision $(\mathrm{CV}, \%)^{\mathrm{a}}$ & 6.9 & 10.3 & 4.3 & 0.80 \\
\hline Accuracy $(\%)^{b}$ & 111 & 100 & 98.9 & 100 \\
\hline
\end{tabular}

The retention times for zabofloxacin and the IS were determined to be approximately 9.67 and 4.08 min, respectively, indicating that the analysis can be completed within 13 min (Fig. 3B and 3C). The retention times for zabofloxacin and IS in the standard bile samples were identical to those of bile samples obtained following oral administration of the drug at a dose of $20 \mathrm{mg} / \mathrm{kg}$.

Under the analytical conditions used in the present study, the calibration curves for zabofloxacin in rat bile were found to be linear over a concentration range of $0.5-100 \mu \mathrm{g} / \mathrm{mL}$. The calibration equation for zabofloxacin was $(n=5$, the slopes and intercepts in the mean \pm standard deviation) $\mathrm{y}=(0.0243 \pm 0.0004) \mathrm{x}-(0.0049 \pm$ 0.0108 ) with $r^{2}=0.9994 \pm 0.0004$, where $y, x$ and $r$ represent the peak area ratio, the concentration of zabofloxacin in the bile, and the correlation coefficient, respectively.

QC samples at 4 different concentrations $(0.5,5$, 25 , and $100 \mu \mathrm{g} / \mathrm{mL}$ ) were analyzed in 5 replicates to determine the intraday and interday accuracy/precision. A summary of the intraday and interday accuracy/precision is listed in Table 2 . The intraday accuracy values for zabofloxacin ranged from 102 to $105 \%$, while the value for intraday precision in RSD was less than $5.3 \%$. The values for interday accuracy for the analyte ranged from 98.9 to $111 \%$, and the precision was less than $10.3 \%$. The limit of detection (LOD) for zabofloxacin was $<0.5 \mu \mathrm{g} / \mathrm{mL}$ for the bile sample. Based on the estimated precision and accuracy, the limit of quantification for this assay was set at 0.5 $\mu \mathrm{g} / \mathrm{mL}$.

The stability of zabofloxacin in the rat bile was assessed for typical storage and handling conditions (Table 4). After freeze-thaw cycles, stabilities of zabofloxacin were $57.7-73.6 \%$ and $6.5-25.1 \%$ at room temperature for $24 \mathrm{~h}$. For long-term stability $\left(-80^{\circ} \mathrm{C}\right.$ for 1 week), the peak ratios were comparable to those of the fresh sample (i.e., 98.0$101 \%)$. 

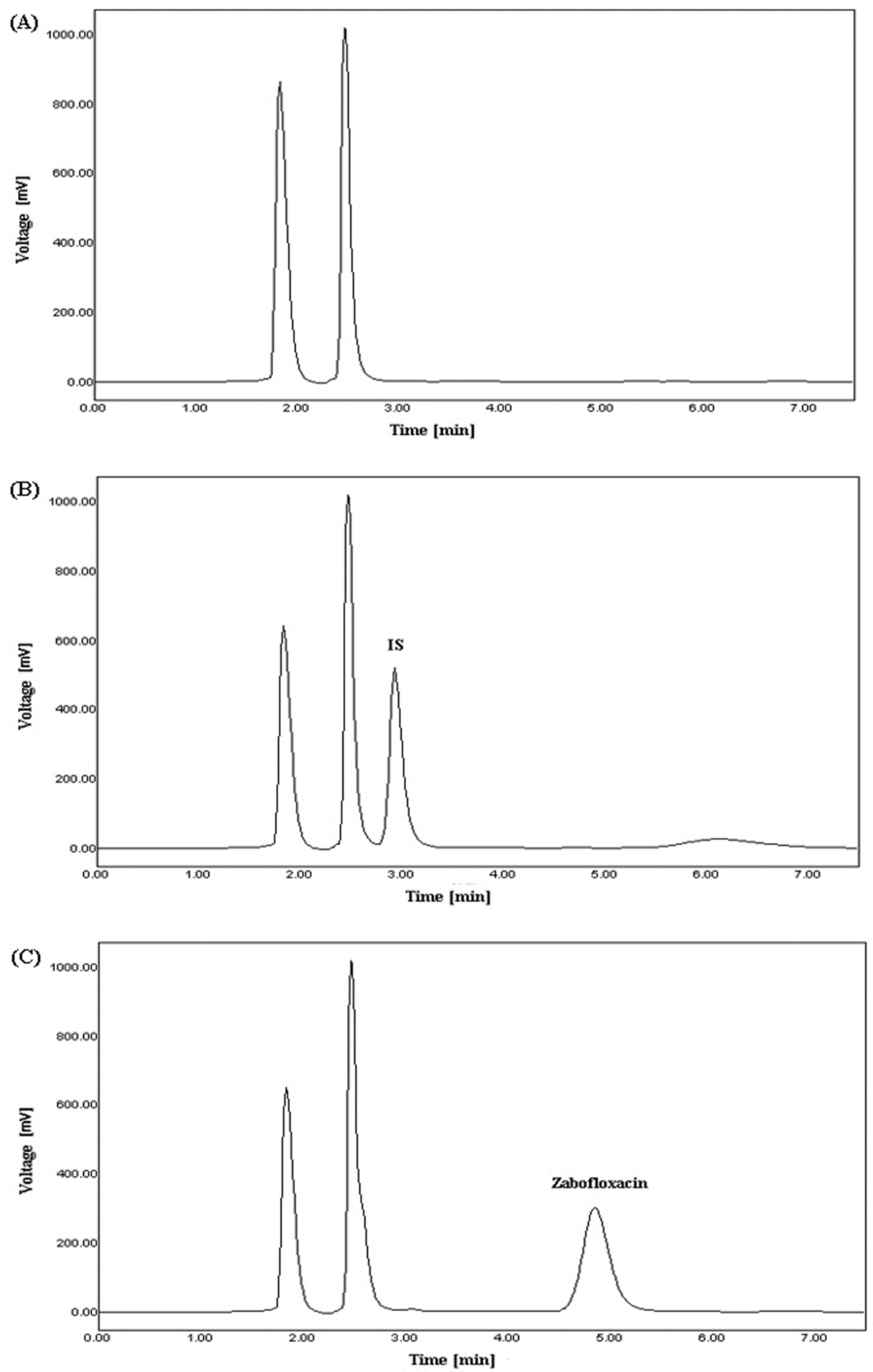

Figure 2. Representative HPLC chromatograms of (A) blank rat plasma, (B) rat plasma spiked with $1 \mu \mathrm{g} / \mathrm{mL}$ of enrofloxacin (i.e. the internal standard), and (C) rat plasma spiked with $5 \mu \mathrm{g} / \mathrm{mL}$ of zabofloxacin. 

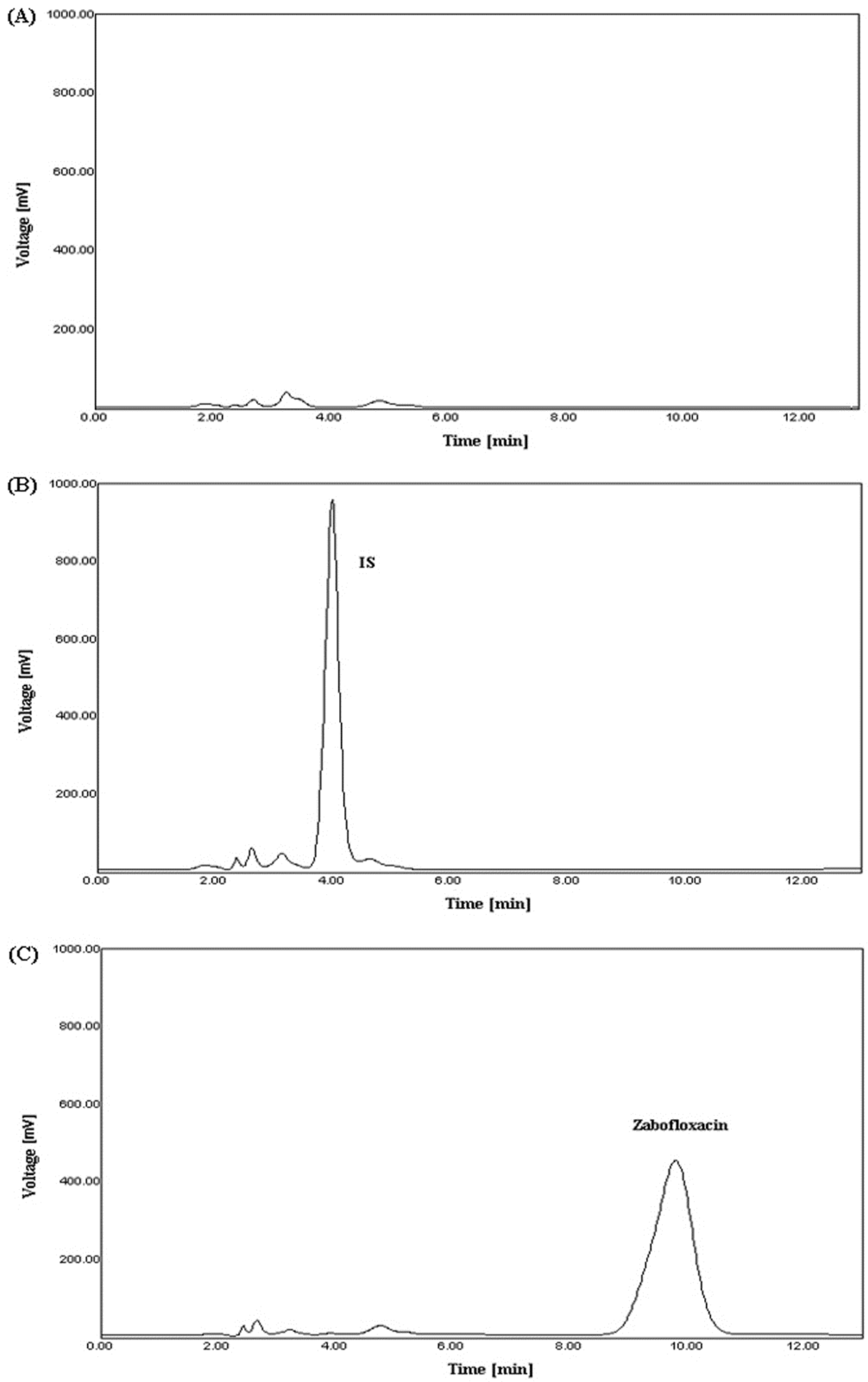

Figure 3. Representative HPLC chromatograms of (A) blank rat bile, (B) rat bile spiked with $15 \mu \mathrm{g} / \mathrm{mL}$ of enrofloxacin (i.e. the internal standard), and (C) rat bile spiked with $50 \mu \mathrm{g} / \mathrm{mL}$ of zabofloxacin. 


\section{Assay Validation of Zabofloxacin in the Urine}

Fig. 4 presents representative HPLC chromatograms of blank rat urine, blank urine spiked with zabofloxacin, and blank urine spiked with enrofloxacin (IS). After optimizing the chromatographic separation conditions, a reproducible separation among zabofloxacin, IS, and endogenous components was obtained. The chromatogram for a blank rat urine sample showed no interfering peaks for the retention times of the analyte and the IS (Fig. 4). The retention times for zabofloxacin and the IS were identical to those of bile (Fig. 4B and 4C). The retention times for zabofloxacin and the IS in the standard urine samples were identical to those of urine samples obtained following oral administration of the drug at a dose of $20 \mathrm{mg} / \mathrm{kg}$.

Calibration curves for zabofloxacin in rat urine were found to be linear over a concentration range of $0.5-100 \mu \mathrm{g} / \mathrm{mL}$. The calibration equation for zabofloxacin was $(n=5$, the slopes and intercepts in the mean \pm standard deviation $) \mathrm{y}=(0.0250 \pm$ $0.0003) \mathrm{x}-(0.0060 \pm 0.0021)$ with $\mathrm{r}^{2}=0.9999 \pm$ 0.0002 , where $y, x, r$ represent the peak area ratio, the concentration of zabofloxacin in the urine, and the correlation coefficient, respectively.

QC samples at 4 different concentrations $(0.5,5$, 25 , and $100 \mu \mathrm{g} / \mathrm{mL}$ ) were analyzed in 5 replicates to determine the intraday and interday accuracy/precision. A summary of the values for intraday and interday precision/accuracy is listed in Table 3. The intraday accuracy values for zabofloxacin ranged from 97.6 to $113 \%$, while the intraday precision in RSD was less than $8.1 \%$. The interday accuracy values for the analyte ranged from 98.5 to $110 \%$, and the precision was less than $7.0 \%$. The limit of detection (LOD) of zabofloxacin was $<0.5 \mu \mathrm{g} / \mathrm{mL}$ for the urine sample. Based on the estimated precision and accuracy, the limit of quantification for this assay was set at $0.5 \mu \mathrm{g} / \mathrm{mL}$.

The stability of zabofloxacin in the rat urine was assessed for typical storage and handling conditions (Table 4). After freeze-thaw cycles, the changes in the ratios of the peak areas of zabofloxacin to those of the IS were negligible (i.e., 98.2-102\%). In the case of bench-top stability, and long-term stability, the peak ratios were comparable to those of the fresh sample (i.e., $97.4-97.7 \%$ for bench-top stability, and $97.0-103 \%$ for long-term stability).

\section{Pharmacokinetics following Intravenous and} Oral Administration of Zabofloxacin to Rats

The applicability of the current assay method to the study of the pharmacokinetics of zabofloxacin in rats was also examined. Fig. 5A shows the temporal profiles of zabofloxacin concentrations in plasma after a single intravenous and oral administration of zabofloxacin at a dose of $20 \mathrm{mg} / \mathrm{kg}$. The concentration of the drug was readily measurable in plasma samples collected up to $8 \mathrm{hr}$ after zabofloxacin administration. The standard pharmacokinetic parameters such as $\mathrm{V}_{\mathrm{ss}}, \mathrm{C}_{\max }, \mathrm{T}_{\max }$, $t_{1 / 2}, A U C_{\infty}, C_{b}$, and $C_{R}$ of zabofloxacin are listed in Table 5. Fig. 5B shows the temporal profiles for the cumulative biliary excretion (amount, mean $\pm \mathrm{SD}$, $\mathrm{n}=4$ ) of zabofloxacin after the oral administration. The cumulative amount of zabofloxacin excreted into bile for $0-24 \mathrm{~h}$ was $7.4 \pm 1.0 \%$ of the treated dose. The urinary recovery of zabofloxacin in $24 \mathrm{~h}$ was $8.0 \pm 4.5 \%$ of the treated dose. This is the first report for the biliary and urinary excretion of zabofloxacin in rats. These collective observations suggest that the current assay is applicable to pharmacokinetic studies of zabofloxacin in rats.

\begin{tabular}{lcccc}
\hline \multicolumn{5}{l}{ Table 3. Precision and accuracy of zabofloxacin in rat urine $(\mathrm{n}=5)$. } \\
\hline Batch & LOQ & Theoretical concentration $(\mu \mathrm{g} / \mathrm{mL})$ & \\
& 0.5 & 5 & MQC & HQC \\
& & & 25 & 100 \\
(A) Intraday accuracy and precision & 0.56 & 4.91 & 24.4 & 98.4 \\
Mean estimated concentration $(\mu \mathrm{g} / \mathrm{mL})$ & 6.6 & 8.1 & 6.3 & 4.6 \\
Precision $(\mathrm{CV}, \%)^{\mathrm{a}}$ & 113 & 98.4 & 97.6 & 98.4 \\
Accuracy $(\%)^{\mathrm{b}}$ & & & & \\
& & & 25.0 & 100 \\
(B) Interday accuracy and precision & 0.55 & 4.9 & 1.6 & 0.30 \\
Mean estimated concentration $(\mu \mathrm{g} / \mathrm{mL})$ & 7.0 & 2.0 & 100 & 100 \\
Precision $(\mathrm{CV}, \%)^{\mathrm{a}}$ & 110 & 98.5 & \\
Accuracy $(\%)^{\mathrm{b}}$ & \multicolumn{4}{c}{} \\
\hline${ }^{\mathrm{a}}$ Precision $(\mathrm{CV}, \%)=$ standard deviation of the concentration / mean concentration $\times 100$. \\
${ }^{\mathrm{b}}$ Accuracy $(\%)=$ calculated concentration / theoretical concentration $\times 100$. \\
\hline
\end{tabular}



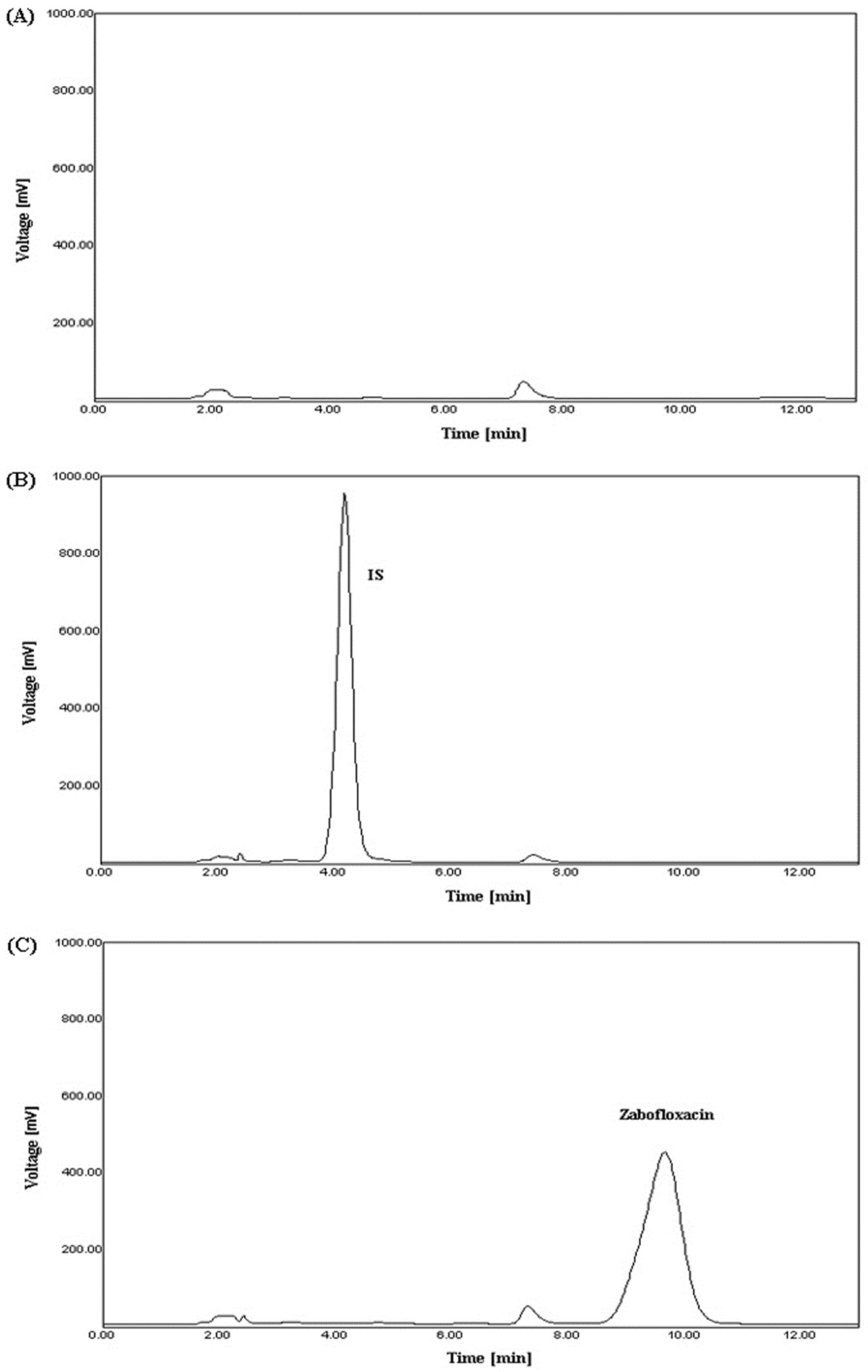

Figure 4. Representative HPLC chromatograms of (A) blank rat urine, (B) rat urine spiked with $15 \mu \mathrm{g} / \mathrm{mL}$ of enrofloxacin (i.e. the internal standard), and (C) rat urine spiked with $50 \mu \mathrm{g} / \mathrm{mL}$ of zabofloxacin. 


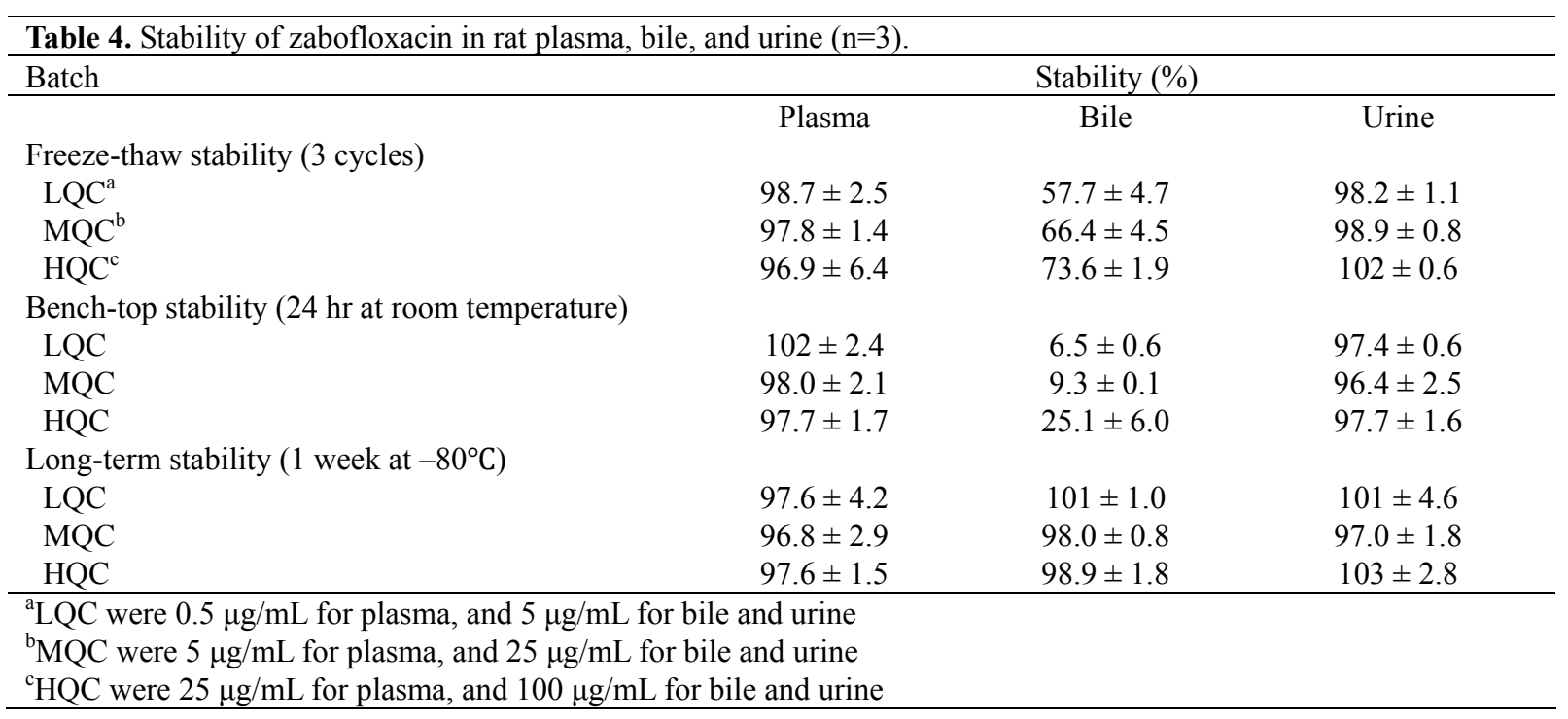

(A)

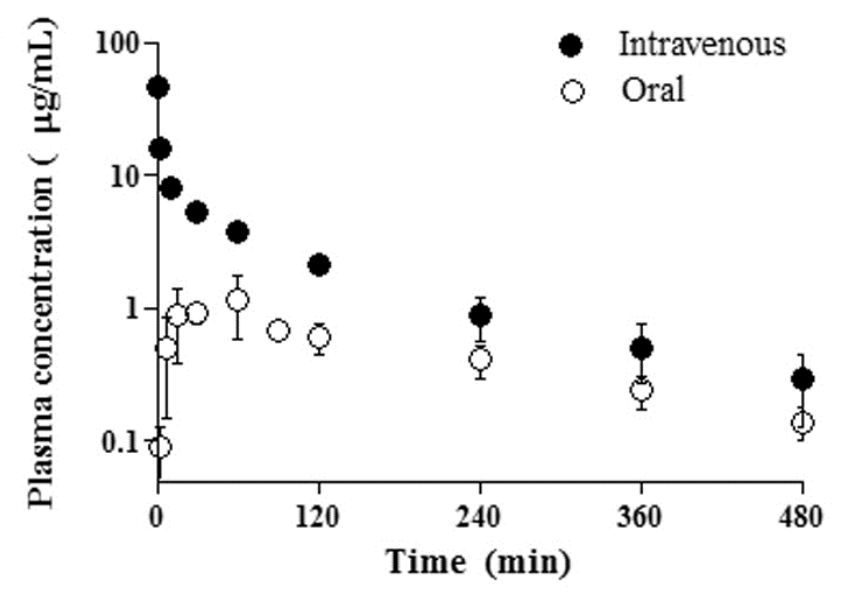

(B)

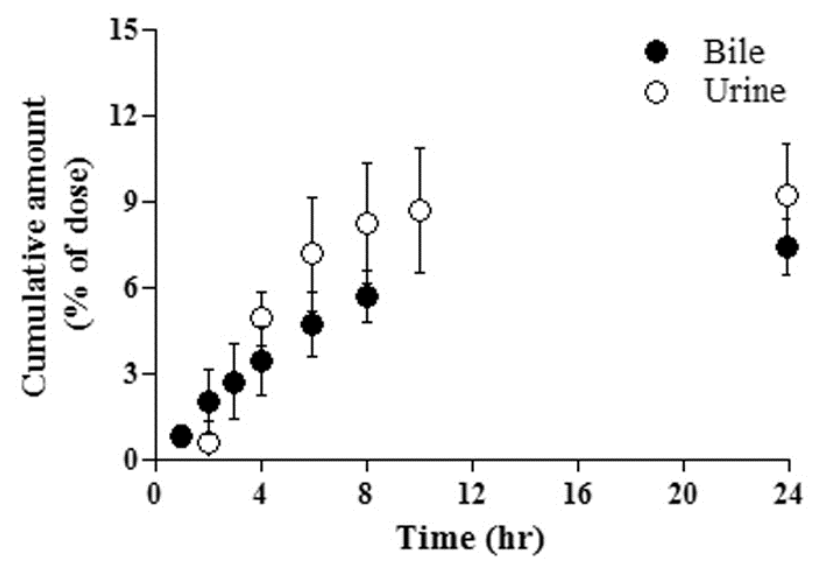

Figure 5. Mean plasma concentration-time profiles after intravenous and oral administration (A), and cumulative amount of excretion into bile and urine after oral administration (B) of zabofloxacin at a dose of $20 \mathrm{mg} / \mathrm{kg}$ zabofloxacin to rats. Each point represents the mean $\pm \mathrm{SD}$ for 4 experiments. 


\begin{tabular}{|c|c|}
\hline Pharmacokinetic parameters & Zabofloxacin \\
\hline \multicolumn{2}{|l|}{ Intravenous administration } \\
\hline $\mathrm{AUC}_{\infty}(\mu \mathrm{g} \cdot \mathrm{min} / \mathrm{mL})$ & $798 \pm 135$ \\
\hline $\mathrm{CL}(\mathrm{mL} / \mathrm{min} / \mathrm{kg})$ & $20.0 \pm 3.2$ \\
\hline $\mathrm{V}_{\mathrm{ss}}(\mathrm{L} / \mathrm{kg})$ & $2.4 \pm 0.3$ \\
\hline \multicolumn{2}{|l|}{ Oral administration } \\
\hline $\mathrm{T}_{\max }(\min )$ & $33.8 \pm 18.9$ \\
\hline $\mathrm{C}_{\max }(\mu \mathrm{g} / \mathrm{mL})$ & $1.4 \pm 0.5$ \\
\hline $\mathrm{T}_{1 / 2}(\min )$ & $169 \pm 36.2$ \\
\hline $\mathrm{AUC}_{\infty}(\mu \mathrm{g} \cdot \mathrm{min} / \mathrm{mL})$ & $221 \pm 23.8$ \\
\hline $\mathrm{CL}_{\mathrm{b}}(\mathrm{mL} / \mathrm{min} / \mathrm{kg})$ & $5.8 \pm 0.8$ \\
\hline $\mathrm{CL}_{\mathrm{R}}(\mathrm{mL} / \mathrm{min} / \mathrm{kg})$ & $6.0 \pm 2.8$ \\
\hline Bioavailability $^{\mathrm{a}}(\%)$ & 27.7 \\
\hline $\begin{array}{l}\text { AUC: total area under the } \\
\text { clearance; } \mathrm{V}_{\mathrm{ss}} \text { : apparent volu } \\
\text { concentration; } \mathrm{T}_{1 / 2} \text { : terminal-p } \\
{ }^{\mathrm{a} B i o a v a i l a b i l i t y ~}(\%) \text { was calcu }\end{array}$ & $\begin{array}{l}\text { e from zero to infinity; CL: total body } \\
\text { ate; } T_{\max } \text { : time to reach } C_{\max } ; C_{\max } \text { : peak } \\
\text { ince; } \mathrm{CL}_{R} \text { : renal clearance } \\
\text { intravenous } A U C_{\infty}\end{array}$ \\
\hline
\end{tabular}

\section{DISCUSSION}

A simple reversed-phase HPLC method using fluorescence detection was developed and validated for the simultaneous determination of zabofloxacin, a novel fluoquinolone antibiotic. The plasma sample was treated by methanol for protein precipitation, and bile and urine sample treatment included deproteinization and extraction of the drug by chloroform. Zabofloxacin extraction in the bile and urine was investigated using general organic solvents such as chloroform, n-hexane and methyl chloride. Chloroform was the most suitable for zabofloxacin analysis among three organic solvents. Representative HPLC chromatograms of a blank, a blank spiked with zabofloxacin, and a blank spiked with enrofloxacin (IS) were presented for plasma, bile, and urine (Fig. 2-4). After optimizing the chromatographic separation conditions, a reproducible separation among zabofloxacin, IS, and endogenous components was obtained. The retention times for zabofloxacin and the IS in plasma were determined to be approximately 5.25 and $3.05 \mathrm{~min}$, respectively, indicating that the analysis can be completed within $7.5 \mathrm{~min}$, which is shorter than the previous LC-MS method (i.e., within $8 \mathrm{~min}$ ) (11). Retention times for the bile and urine samples (i.e., 9.67 and $4.08 \mathrm{~min}$, for zabofloxacin and the IS, respectively) were longer than that for the plasma sample in order to avoid interference with other peaks (i.e., endogenous compounds). The retention times for zabofloxacin and IS in the standard samples were identical to those of pharmacokinetics samples obtained following intravenous and oral administration of the drug at a dose of $20 \mathrm{mg} / \mathrm{kg}$.

Response of HPLC with fluorescence is directly proportional to the concentration of zabofloxacin in biological fluids. Under the analytical conditions used in the present study, the calibration curves for zabofloxacin were found to be linear over a concentration range of $0.05-25 \mu \mathrm{g} / \mathrm{mL}$ for plasma and $0.5-100 \mu \mathrm{g} / \mathrm{mL}$ for bile and urine. As compared with a previous study by LC-MS analysis, the calibration curves for zabofloxacin in rat plasma were found to be linear over a concentration range of $10-5000 \mathrm{ng} / \mathrm{mL}$ (11), the sensitivity of LC-MS was 5 times more sensitive than that of LCfluorescence. Summaries of the intraday and interday accuracy/precision values of QC samples are listed in Tables 1-3. These results indicated that the accuracy and precision of the current assay were within the recommendations for assay validation as stipulated in "Guidance for Industry: Bioanalytical Method Validation (12)," and that the reproducibility of the assay was adequate.

The stability of zabofloxacin in the rat biological samples was assessed for typical storage and handling conditions (i.e., 3 freeze-thaw cycles, $24 \mathrm{~h}$ at room temperature, and 1 week at $-80^{\circ} \mathrm{C}$, 
Table 4). Zabofloxacin and the IS were stable in the plasma and urine following storage under typical conditions of processing and sample handling. Zabofloxacin in bile was stable in long-term stability testing $\left(-80^{\circ} \mathrm{C}\right.$ for 1 week). However, zabofloxacin in bile was not stable after $24 \mathrm{~h}$ at room temperature and repeated freeze-thaw cycles (6.5-25.1\% and 57.7-73.6\%, respectively). Therefore, Due to limited stability of zabofloxcin in rat bile, freeze-thaw cycles or prolonged handling in room temperature conditions is not recommended.

The applicability of the current assay method to the study of the pharmacokinetics of zabofloxacin in rats was also examined. The temporal profiles of zabofloxacin concentrations in plasma after a single intravenous and oral administration of zabofloxacin at doses of $20 \mathrm{mg} / \mathrm{kg}$ are shown in Fig. 5A. Plasma concentrations of zabofloxacin at $12 \mathrm{~h}$ in rats were lower than the limit of detection (LOD) and extrapolation of $\mathrm{AUC}$ (i.e., $\mathrm{AUC}_{8-\infty}$ ) was no more than $20 \%$ of $\mathrm{AUC}_{\infty}$. Blood sampling until $8 \mathrm{~h}$ was sufficient for pharmacokinetic study of zabofloxacin. Pharmacokinetic parameters following oral administration of zabofloxacin were comparable to reported values (11). After oral administration of zabofloxacin in rats, absorption of zabofloxacin was quite fast, and $\mathrm{T}_{\max }$ (time to reach $\mathrm{C}_{\max }$ ) was about $30 \mathrm{~min}$ (Table 5). The drug absorption was also fast in the previous pharmacokinetics of dogs with a $\mathrm{T}_{\max }$ of 1-3 $\mathrm{h}$ (15). Bioavailability (\%) of zabofloxacin in rats, calculated by dividing oral $\mathrm{AUC}_{\infty}$ by intravenous $\mathrm{AUC}_{\infty}$, was $27.7 \%$ (Table 5). Bioavailability of zabofloxacin was quite low by comparison with another fluoroquinolone, ofloxacin $(78 \%$ in rats)(16). However, drug absorption that is low in rats may not necessarily be low in humans. Although bioavailability of ciprofloxacin in rats was about $17 \%$, in humans it was $69 \%$ (17-18). After the oral administration of zabofloxacin, biliary clearance $\left(\mathrm{CL}_{\mathrm{b}}\right)$ and renal clearance $\left(\mathrm{CL}_{\mathrm{R}}\right)$ were similar, $5.8 \pm 0.8$ and $6.0 \pm 2.8 \mathrm{~mL} / \mathrm{min} / \mathrm{kg}$, respectively (Fig. 5B and Table 5).

Zabofloxacin was excreted into bile and urine at about 7 and 9\% each per oral administration (Fig. 5B). The most common adverse effects associated with fluoroquinolones are gastrointestinal (GI) disturbances such as nausea, vomiting, and diarrhea, skin disturbances, and CNS effects including headaches and dizziness (19). The severity of toxic effects varies with the individual fluoroquinolone and the dose (20). The adverse events in patients treated with fluoroquinolones are headache, nausea, diarrhea, taste perversion, dizziness, phototoxicity, and skin reactions (21). In the previous study (15), the absolute toxic dose of zabofloxacin was considered to be $30 \mathrm{mg} / \mathrm{kg} / \mathrm{d}$, and no observed adverse effect level was considered to be 10 $\mathrm{mg} / \mathrm{kg} / \mathrm{d}$ for either male or female dogs. Zabofloxacin was found to cause vomiting and might change the physiological condition of the GI tract, and $T_{\max }$ at 30 and $90 \mathrm{mg} / \mathrm{kg} / \mathrm{d}$ was delayed compared with that of a single administration, which caused moderate vomiting (15). In this study, identical doses of zabofloxacin (i.e., $20 \mathrm{mg} / \mathrm{kg}$ ) were administered intravenously and orally to rats to examine the bioavailability of the drug. Although intravenous administration of zabofloxacin at a dose of $20 \mathrm{mg} / \mathrm{kg}$ is a high dose, side effects were not apparent in rats. These collective observations suggest that the current assay is applicable to pharmacokinetic studies of zabofloxacin in rats.

\section{CONCLUSIONS}

A method that uses HPLC with fluorescence was developed and validated for the determination of zabofloxacin in rat plasma, bile and urine following oral and intravenous administration to rats at a dose of $20 \mathrm{mg} / \mathrm{kg}$. Zabofloxacin in plasma samples could be determined over a concentration range of 0.05 $25 \mu \mathrm{g} / \mathrm{mL}$. The method was also applicable to bile and urine samples for concentrations ranging from $0.5-100 \mu \mathrm{g} / \mathrm{mL}$, respectively. Advantages of this method are a simple sample preparation and a short LC run time. In addition, the sensitivity, selectivity, linearity, accuracy, and precision of the assay were adequate, as was the stability of the compound during the assay, with the exception of bile. This method should be applicable to routine assays in pharmacokinetic studies of zabofloxacin in small animals. 


\section{ABBREVIATIONS}

AUC, area under the plasma concentration curve; $\mathrm{AUC}_{\infty}$, total area under the plasma concentrationtime curve from time zero to infinity; $\mathrm{C}_{\max }$, peak plasma concentration; CL: total body clearance; $\mathrm{CL}_{\mathrm{b}}$ : biliary clearance; $\mathrm{CL}_{\mathrm{R}}$ : renal clearance; $\mathrm{HQC}$, high-quality control; $\lambda$, slope of the log-linear portion of the concentration time profile; MQC, mid-quality control; LOQ, limit of quantification; LQC, low-quality control; QC, quality control; $\mathrm{RSD}$, relative standard deviation; $\mathrm{T}_{1 / 2}$, terminal phase half-life; $\mathrm{T}_{\max }$, time to reach; $\mathrm{V}_{\mathrm{ss}}$, apparent volume of distribution at steady state

\section{ACKNOWLEDGEMENT}

This work was supported by the Korea Science and Engineering Foundation (KOSEF) through the National Research Laboratory. The program is funded by the Ministry of Science and Technology (No.R0A-2006-000-10190-0).

\section{REFERENCES}

1. Emmerson AM, Jones AM. The quinolones: decades of development and use. J Antimicrob Chemother, 2003; 51 Suppl 1:13-20.

2. Appelbaum PC, Hunter PA. The fluoroquinolone antibacterials: past, present and future perspectives. Int J Antimicrob Agents, 2000; 16:5-15.

3. Garcia-Garrote F, Cercenado E, Martin-Pedroviejo J, Cuevas O, Bouza E. Comparative in vitro activity of the new quinolone gemifloxacin (SB-265805) with other fluoroquinolones against respiratory tract pathogens. J Antimicrob Chemother, 2001; 47:681-4.

4. Klepser ME, Ernst EJ, Petzold CR, Rhomberg P, Doern GV. Comparative bactericidal activities of ciprofloxacin, clinafloxacin, grepafloxacin, levofloxacin, moxifloxacin, and trovafloxacin against Streptococcus pneumoniae in a dynamic in vitro model. Antimicrob Agents Chemother, 2001; 45:6738.

5. Park HS, Jung SJ, Kwak JH, Choi DR, Choi EC. DNA gyrase and topoisomerase IV are dual targets of zabofloxacin in Streptococcus pneumoniae. Int $\mathrm{J}$ Antimicrob Agents, 2010; 36:97-8.

6. Kim HJ, Seol MJ, Park HS, Choi DR, Seong SK,
Shin HK, Kwak JH. Antimicrobial activity of DW224a, a new fluoroquinolone, against Streptococcus pneumoniae. J Antimicrob Chemother, 2006; 57:1256-8.

7. Park HS, Kim HJ, Seol MJ, Choi DR, Choi EC, Kwak JH. In vitro and in vivo antibacterial activities of DW-224a, a new fluoronaphthyridone. Antimicrob Agents Chemother, 2006; 50:2261-64.

8. Hendershot EF. Fluoroquinolones. Infect Dis Clin North Am, 1995; 9:715-30.

9. Jones RN, Biedenbach DJ, Ambrose PG, Wikler MA. Zabofloxacin (DW-224a) activity against Neisseria gonorrhoeae including quinolone-resistant strains. Diagn Microbiol Infect Dis, 2008; 62:110-2.

10. Theuretzbacher U. Future antibiotics scenarios: is the tide starting to turn? Int J Antimicrob Agents, 2009; 34:15-20.

11. Jin HE, Lee KR, Kang IH, Chung SJ, Shim CK. Determination of zabofloxacin in rat plasma by liquid chromatography with mass spectrometry and its application to pharmacokinetic study. J Pharm Biomed Anal, 2011; 54:873-7.

12. USFDA. U.S. Department of Health \& Human Services, Food and Drug Administration, Guidance for Industry, Bioanalytical Method Validation. 2001.

13. Jin HE, Hong SS, Choi MK, Maeng HJ, Kim DD, Chung SJ, Shim CK. Reduced antidiabetic effect of metformin and down-regulation of hepatic Oct1 in rats with ethynylestradiol-induced cholestasis. Pharm Res, 2009; 26:549-59.

14. Gibaldi M, Perrier D. Pharmacokinetics, 2nd revised and expanded,. Informa HealthCare, New York, 1982:409-16.

15. Han J, Kim JC, Chung MK, Kim B, Choi DR. Subacute toxicity and toxicokinetics of a new antibiotic, DW-224a, after single and 4-week repeated oral administration in dogs. Biol Pharm Bull, 2003; 26:832-9.

16. Wang H, Liao ZX, Chen M, Hu XL. Effects of hepatic fibrosis on ofloxacin pharmacokinetics in rats. Pharmacol Res, 2006; 53:28-34.

17. Nouaille-Degorce B, Veau C, Dautrey S, Tod M, Laouari D, Carbon C, Farinotti R. Influence of renal failure on ciprofloxacin pharmacokinetics in rats. Antimicrob Agents Chemother, 1998; 42:289-92.

18. Drusano GL, Standiford HC, Plaisance K, Forrest A, Leslie J, Caldwell J. Absolute oral bioavailability of ciprofloxacin. Antimicrob Agents Chemother, 1986; 
30:444-6.

19. Janknegt R. Fluoroquinolones. Adverse reactions during clinical trials and postmarketing surveillance. Pharm Weekbl Sci, 1989; 11:124-7.

20. Kashida Y, Kato M. Toxic effects of quinolone antibacterial agents on the musculoskeletal system in juvenile rats. Toxicol Pathol, 1997; 25:635-43.

21. Segev S, Yaniv I, Haverstock D, Reinhart H. Safety of long-term therapy with ciprofloxacin: data analysis of controlled clinical trials and review. Clin Infect Dis, 1999; 28:299-308. 